\title{
Schulische Integration und Wohlbefinden von Kindern und Jugendlichen mit besonderem Förderbedarf
}

\author{
Martin Venetz \\ Hochschule für Heilpädagogik Zürich
}

Die Frage, ob die schulische Integration von Kindern und Jugendlichen mit besonderem Förderbedarf zu ihrem Wohle gereicht, wird nach wie vor sehr kontrovers diskutiert. Um eine Antwort darauf geben zu können, ist zuerst zu klären, was unter Kindeswohl eigentlich zu verstehen ist. Eine grundlegende Differenzierung bezieht sich auf die Unterscheidung zwischen „objektivem Wohlbefinden“ und „subjektivem Wohlbefinden", wie sie beispielsweise in der dritten internationalen UNICEF-Vergleichsstudie (2013) vorgenommen wird: Das objektive Wohlbefinden setzt sich aus fünf Dimensionen zusammen (materielles Wohlbefinden, Gesundheit und Sicherheit, Bildung, Verhalten und Risiken, Wohnen und Umwelt), die nicht nur die jetzigen Lebensumstände, sondern auch Entwicklungsperspektiven - das Wohlergehen - in den Blick nehmen. Im Gegensatz dazu bezieht sich das subjektive Wohlbefinden, das selbst wahrgenommene Ausmaß an Zufriedenheit oder, allgemeiner formuliert, an positiver Befindlichkeit, viel stärker auf das Hier und Jetzt. Die eingangs aufgeworfene Frage wäre demnach sowohl aus einer Außen- (objektives Wohlbefinden) als auch aus einer Innenperspektive (subjektives Wohlbefinden) zu klären.

Obwohl die Befundlage zum objektiven Wohlbefinden von Kindern und Jugendlichen mit besonderem Förderbedarf in der Integration bzw. Separation in der Schweiz eher schmal ist und somit (noch) keine abschließende Ant- wort zulässt, seien zwei - für das objektive Wohlbefinden - bedeutsame Erkenntnisse aus aktuellen, größeren Studien herausgegriffen: Eine im Rahmen des in den 1980er-Jahren initiierten Forschungsprogramms INTSEP um Urs Haeberlin an der Universität Freiburg/ Schweiz durchgeführte Untersuchung hat ergeben, dass Schülerinnen und Schüler mit Schulleistungsschwächen, die integrativ beschult worden sind, anspruchsvollere nachobligatorische Ausbildungsgänge absolvieren und ihnen der Einstieg ins Erwerbsleben deutlich besser gelingt (Eckhart u. a. 2011). Ergebnisse von Müller u. a. (2012) zu Effekten der Klassenzusammensetzung werden dahingehend diskutiert, dass integrative Schulungsformen möglicherweise einen positiven Effekt auf Verhaltenskompetenzen von Jugendlichen mit Förderbedarf im emotional-sozialen Bereich haben könnten.

Wie sieht nun die Innenperspektive - das subjektive Wohlbefinden - von Kindern und Jugendlichen mit besonderem Förderbedarf in der Integration bzw. Separation aus? Hierzu muss die Bemerkung vorangeschickt werden, dass das subjektive (emotionale) Wohlbefinden in der Schule eine wesentliche pädagogische Zielvorstellung darstellt und in den Leitgedanken von Schulen einen zentralen Stellenwert einnimmt. So ist beispielsweise im Leitbild einer Primarschule zu lesen: „Unsere Schule ist ein Ort, wo ich mich wohlfühle." Für Haeber- 
lin u. a. $(1989,13)$ ist das subjektive Wohlbefinden von Kindern und Jugendlichen mit besonderem Förderbedarf in der Schule gar einer der "zuverlässigsten Indikatoren für gelingende oder misslingende pädagogische Integration“. Die empirische Befundlage für die Schweiz hierzu ist sehr dürftig und zudem wenig konsistent. Während Haeberlin u. a. (1991) im Rahmen ihrer INTSEP-Studie zum Schluss kommen, dass die schulische Integration von Kindern und Jugendlichen mit Lernbehinderungen ihrem subjektiven Wohlbefinden in der Schule abträglich ist, sind in der Studie von Venetz u. a. (2012) keine bedeutsamen Unterschiede zwischen Schülerinnen und Schülern mit und ohne Schulleistungsschwächen in Regelklassen festzustellen. (Lernende mit Verhaltensauffälligkeiten fühlen sich in der Schule - unabhängig von der Schulungsform - emotional generell weniger wohl.)

In den bisherigen Ausführungen zum subjektiven Wohlbefinden sind zwei bedeutsame Differenzierungen noch nicht angesprochen worden. Die erste betrifft den Unterschied zwischen dem habituellen und dem aktuellen Wohlbefinden, das heißt zwischen der überdauernden Grundstimmung (in der Schule) auf der einen und dem aktuellen Befinden in einer konkreten (Unterrichts-)Situation auf der anderen Seite. Dass diese beiden Aspekte nicht dasselbe sind, ist offensichtlich: Auch Schülerinnen und Schüler, die sich im Allgemeinen in der Schule sehr wohl fühlen, langweilen sich ab und an im Unterricht oder sind unzufrieden. Umgekehrt lassen sich auch Kinder und Jugendliche, die nicht gerne zur Schule gehen, für bestimmte Lerninhalte leicht begeistern. Das aktuelle Wohlbefinden resultiert also immer aus dem Zusammenspiel von Person und Situation sowie deren Interaktion. Es fluktuiert über die Zeit viel stärker als das habituelle Wohlbefinden. Die zweite Differenzierung ist grundsätzlicher und beruht auf der Einsicht, dass Wohlbefinden nicht nur Befindenszustände positiv erlebter Deaktivierung wie ,ausge- glichen“, „unbesorgt“ oder „entspannt“ umfasst, sondern auch solche positiv erlebter Aktivierung wie „begeistert", „,interessiert" oder „hoch konzentriert“. Für ein ganzheitliches Verständnis aktuellen Wohlbefindens sind deshalb beide Formen positiven Erlebens gleichzeitig und gleichwertig zu berücksichtigen.

Über das aktuelle subjektive Wohlbefinden von integriert beschulten Schülerinnen und Schülern mit besonderem Förderbedarf weiß man bislang nur sehr wenig. Die Studie von Venetz u.a. (2012) zeigt, dass sich integriert beschulte Schülerinnen und Schüler mit Schulleistungsschwächen aktiv und interessiert am Unterrichtsgeschehen beteiligen und nicht wie immer wieder vermutet - völlig überfordert sind. Auch Lernende mit Verhaltensauffälligkeiten berichten im Vergleich zu ihren Mitschülerinnen und -schülern nicht über weniger Befindenszustände positiver Aktivierung; allerdings erleben sie mehr negative Befindenszustände wie Stress, Ärger oder Nervosität. Einen entscheidenden Einfluss auf das aktuelle Wohlbefinden haben auch Gegebenheiten der jeweiligen Unterrichtssituation, was nicht zuletzt auf die Bedeutung der Unterrichtsgestaltung hinweist.

Die in knapper Form ausgeführten Gedanken machen vor allem eines klar: Obwohl viel über (die Bedeutung der Schulungsform für) das Wohlbefinden von Kindern und Jugendlichen mit besonderem Förderbedarf gesprochen wird, wissen wir eigentlich nur sehr wenig darüber. Führt man sich die Bedeutung positiven Befindens - und zwar vor allem desjenigen, das mit hoher Aktivierung verknüpft ist - für ein erfülltes und glückliches Leben vor Augen (s. dazu beispielsweise die Metaanalyse von Lyubomirsky u. a. 2005), so wäre zu wünschen, dass sich auch die Heil- und Sonderpädagogik vermehrt mit dem aktuellen Wohlbefinden von Schülerinnen und Schülern mit besonderem Förderbedarf in ihrem unmittelbaren Lebensalltag beschäftigt. 


\section{Literatur}

Eckhart, M.; Haeberlin, U.; Sahli Lozano, C.; Blanc, P. (2011): Langzeitwirkungen der schulischen Integration. Bern: Haupt

Haeberlin, U.; Moser, U.; Bless, G.; Klaghofer, R. (1989): Integration in die Schulkasse. Fragebogen zur Erfassung von Dimensionen der Integration von Schülern FDI 4-6. Bern: Haupt Haeberlin, U.; Bless, G.; Moser, U.; Klaghofer, R. (1991): Die Integration von Lernbehinderten. Versuche, Theorien, Forschungen, Enttäuschungen, Hoffnungen. Bern: Haupt

Lyubomirksy, S.; King, L.; Diener, E. (2005): The benefits of frequent positive affect: Does happiness lead to success? In: Psychological Bulletin 131, 803-855. http://dx.doi.org/10.1037/oo 33-2909.131.6.803

Müller, C. M.; Hofmann, V.; Studer, F. (2012): Lässt sich individuelles Problemverhalten durch das Niveau an Verhaltensschwierigkeiten unter den Mitschülern vorhersagen? Ergebnisse einer Querschnittsstudie und ihre Relevanz für die Frage einer integrativen vs. separativen Beschulung verhaltensauffälliger Schüler. In: Empirische Sonderpädagogik 4, 111-128

UNICEF Office of Research (2013): Child wellbeing in rich countries: A comparative overview (Innocenti Report Card 11). Florenz: UNICEF Office of Research

Venetz, M.; Tarnutzer, R.; Zurbriggen, C.; Sempert, W. (2012): Emotionales Erleben im Unterricht und schulbezogene Selbstbilder. Bern: SZHI CSPS

\section{Anschrift des Autors}

\section{Dr. phil. Martin Venetz}

Interkantonale Hochschule für Heilpädagogik HfH

Schaffhauserstr. 239

Postfach 5850

$\mathrm{CH}-8050$ Zürich

Tel.: +41 (o) 443171154

E-Mail: martin.venetz@hfh.ch 